\title{
Optimization of Slope Critical Surfaces Considering Seepage and Seismic Effects Using Finite Element Method and Five Meta-Heuristic Algorithms
}

\author{
Ali Kaveh ${ }^{1 *}$, Mohammad Reza Seddighian ${ }^{1}$ \\ ${ }^{1}$ School of Civil Engineering, Iran University of Science and Technology, Narmak, Tehran, Postal Code 16846-13114, Iran \\ * Corresponding author, e-mail: alikaveh@iust.ac.ir
}

Received: 27 August 2020, Accepted: 02 December 2020, Published online: 09 December 2020

\begin{abstract}
One of the most crucial geotechnical engineering problems is the stability of slopes that are still attracting scientists and engineers. In this study, five recently developed meta-heuristic methods are utilized to determine the Critical Failure Surface (CFS) and its corresponding Factor of Safety (FOS). Through the FOS calculations, the Finite Element Method (FEM) is employed to convert the strong form of the main differential equation to a weak form. Additional to the general loading, seismic forces and seepage effect are considered, as well. Finally, the proposed optimization procedure is applied to numerical benchmark examples, and results are compared with other methods.
\end{abstract}

Keywords

optimization, Meta-Heuristic Algorithm, Enriched Firefly Algorithm, Black Hole Mechanics Optimization, Finite Element Method, slope stability, Critical Failure Surface, soil mechanics, seepage, seismic analysis

\section{Introduction}

One of the most crucial geotechnical engineering problems is the stability of slopes that are still attracting scientists and engineers. Over the years, the analysis of this problem has advanced from tedious manual calculations to high-level computer algorithms. Hence, the researcher's comprehension of the stability of slopes has improved due to the ameliorate of computational methods. In the slope stability problems, one of the critical aims is to evaluate the Factor of Safety (FOS) corresponding with the critical failure surface of the slope. Usually, the evaluation of FOS is executed by widely popular Limit Equilibrium Techniques (LETs). There are several well-known and efficient LET, such as Fellenius [1], Bishop and Morgenstern [2], Morgenstern and Price [3], and Spencer [4] to estimate the FOS of slopes against failure.

A complete slope stability analysis requires investigation of the Critical Failure Surface (CFS) corresponding to the minimum FOS among all probable Trial Failure Surfaces (TFS). There are some traditional methods, such as the grid search method, to detect a CFS. Also, some researchers, such as Baker and Garber [5], Chen and Shao [6], Celestino and Arai and Tagyo [7], He et al. [8] and Varga and Czap [9], have utilized classical optimization procedures. Examples of these methods are variation, simplex method, and conjugate-gradient method to calculate the minimum FOS. Although these conventional methods are robust, straight forward, and swift, however, it is possible to get trapped to a local minimum due to consideration of a smaller number of trial failure surfaces. On the other hand, by considering more TFS, the search procedure to find the CSF will be impossible due to run time and allocated computer memory error. To overcome the mentioned drawbacks of the classical optimization methods, it is possible to utilize MetaHeuristic algorithms.

Nowadays, meta-heuristic algorithms have found many applications in different fields of applied mathematics, engineering, medicine, economics, and other sciences, Kaveh [10]. It is possible to obtain optimal or near-optimal solutions to the severe and even NP-complete problems within an affordable computational time using meta-heuristic algorithms, Coello et al. [11]. They generally mimic a complicated or simple approach to investigate the space of solutions without consuming much computational costs. The mentioned and many other advantages encouraged researchers to employ meta-heuristic algorithms as optimizer to different complicated optimization problems. 
Animals' behavior such as flocking, migrating, hunting, and foraging approaches can be studied and be employed as swarm intelligent rules for developing efficient meta-heuristic algorithms. For example, the robust algorithm that is known as Particle Swarm Optimization (PSO) is inspired by the social behavior of fish schooling or birds flocking [12]. Ant Colony Optimization (ACO) algorithm is developed by observing the pheromone-based communication strategy of biological ants [13]. Firefly Algorithm (FA) and its Enriched version [14] are proposed according to the luminary flashing activities of fireflies to attract the partners in risk warning.

Some of the meta-heuristic algorithms employ the biological evolution concepts, such as mutation, crossover, and natural selection. These types of methods are called Evolutionary Algorithms (EAs), and Genetic Algorithm (GA), Evolution Strategy (ES) algorithm, evolutionary programming (EP), and Genetic Programming (GP) are the most famous instances in this category [15], and [16]. Also, meta-heuristics can be developed based on physical laws such as Colliding Bodies Optimization (CBO) algorithm [17], Thermal Exchange Optimization algorithm [18], and Black Holes Mechanics Optimization (BHMO) algorithm [19].

Another type of meta-heuristics are some algorithms having no clear origin, and some of them are based on mathematical models. The Covariance Matrix Adaptation Evolution Strategy (CMA-ES) algorithm [20], Eigenvectors of the Covariance Matrix (ECM) algorithm [21], and Sine Cosine Algorithm (SCA) can be categorized as this group of algorithms. Moreover, some methods, such as BioGeography-Based Optimization (BBO) algorithm [22], maybe classified in more than one category.

As seen, many methods have been established as meta-heuristic algorithms. Each one is successful in one or several search patterns and optimization problem types. This fact can be deducted from the No Free Lunch (NFL) theorem, which states that there is no universal, robust algorithm for all types of problems [23]. Therefore, studying the new patterns, social behavior, etc., for developing new robust algorithms are required.

In this study, five novel meta-heuristic methods, including Black Hole Mechanics Optimization (BHMO), Enriched Firefly Algorithm (EFA), Eigenvectors of the Covariance Matrix (ECM), Covariance Matrix Adaptation Evolution Strategy (CMA-ES), and Sine Cosine Algorithm (SCA), are utilized to determine critical failure surface due to reduction of the FOS. The paper reports the outcomes of the mentioned algorithms in solving homogenous soil slope, layered slope considering the effect of the phreatic surface resulting from steady-state seepage, and seismic analysis. To obtain more reliable analysis, the Finite Element Method (FEM) concepts are employed to convert the strong form of Richard's differential equation to the weak form. As a comparative study in meta-heuristic method robustness, the final results of the employed algorithms are compared together. Also, for validation, the results of the current study have been verified by already available published results of literature, such as [24-25]. Some relevant papers on reliability can be found in Movahedi Rad [26], Lógó et al. [27] and Kaveh et al. [28], Tauzowski et al. [29], Blachowski et al. [30].

The rest of this paper is organized as follows. Section 2 is dedicated to the main differential equation of the fluid flow within a porous medium, its strong form, weak form, and finite element formulation. In Section 3, the utilized meta-heuristic algorithms have been introduced in a nutshell. Section 4 represents numerical examples and comparative deductions of employed algorithm robustness. Finally, Section 5 concludes the results of the current study.

\section{Fluid flow equations through a porous medium}

In many real-world engineering problems, it is necessary to model fluid flow through a porous medium such as the flow of water through soil, earthen dam, and through pipes or around solid bodies. By some considerations, their form of basic differential equations is alike. This section is dedicated to develop and present the basic formulation of fluid flow analysis in a porous medium. Firstly, the strong form of the central equation is performed, and then its weak form is developed for the finite element analysis. In the procedure of establishing the equations, the fluid is considered as an ideal one in a steady-state, not rotating, incompressible, and inviscid.

\subsection{The Strong-Form Formulation}

To derive the basic differential equation of the fluid flow through a porous medium, firstly, a one-dimensional control volume is considered. Then it is extended to two-dimensional problems. Fig. 1 illustrates a control volume for one-dimensional fluid flow.

According to the volume control illustrated in Fig. 1, Eq. (1) can be stated based on the conservation of mass.

$M_{\text {in }}+M_{b}=M_{\text {out }}$,

where $M_{i n}$ is the mass entering the control volume, $M_{b}$ is the mass generated within the body, and $M_{\text {out }}$ is the mass leaving the control volume, all in units of kilograms or slugs. 


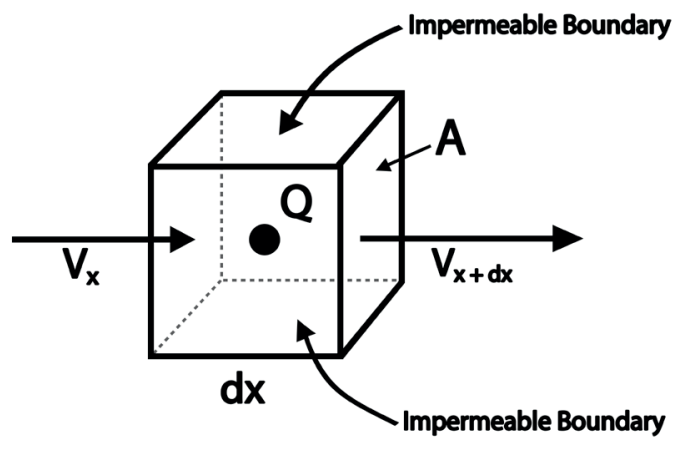

Fig. 1 Control volume for one-dimensional fluid flow

It is possible to restate Eq. (1) in the form of Eq. (2).

$\rho v_{x} A d t+\rho Q d t=\rho v_{x+d x} A d t$,

where $v_{x}$ is the velocity of the fluid flow at surface edge $x$, in units of $\mathrm{m} / \mathrm{s}$ or in./s. $v_{x+d x}$ is the velocity of the fluid leaving the control volume at surface edge $x+d x$. $t$ is time, in unit of second. $Q$ is an internal volumetric flow rate, in $\mathrm{m}^{3} / \mathrm{s}$ or in. ${ }^{3} / \mathrm{s}$. $\rho$ is the mass density of the fluid, in $\mathrm{kg} / \mathrm{m}^{3}$ or slug/in. ${ }^{3}$. Finally, $A$ is the cross-sectional area perpendicular to the fluid flow, in $\mathrm{m}^{2}$ or in. ${ }^{2}$.

To relate the velocity of fluid flow to the hydraulic gradient, the change in the fluid head with respect to $x$, Darcy's law can be employed, as stated in Eq. (3).

$v_{x}=-K_{x x} \frac{d \phi}{d x}=-K_{x x} g_{x}$,

where $K_{x x}$ is the permeability coefficient of the porous medium in the x-direction, in $\mathrm{m} / \mathrm{s}$ or in./s. $\varphi$ is the fluid head in $\mathrm{m}$ or in. Lastly, $g_{x}$ is the fluid hydraulic gradient or head gradient.

Eq. (3) states that the velocity in the $x$-direction is proportional to the gradient of the fluid head in the same direction. By using Fourier's law, Eq. (4) can be stated.

$v_{x+d x}=-\left.K_{x x} \frac{d \phi}{d x}\right|_{x+d x}$

By Taylor series expansion, Eq. (5) can be obtained.

$$
v_{x+d x}=-\left[K_{x x} \frac{d \phi}{d x}+\frac{d}{d x}\left(K_{x x} \frac{d \phi}{d x}\right) d x\right]
$$

where the expansion is truncated by the two-term.

By substituting Eqs. (3) and (5) into Eq. (2), dividing Eq. (2) by $\rho A d x d t$, and simplifying, the basic differential equation for one-dimensional problems can be stated as Eq. (6).

$\frac{d}{d x}\left(K_{x x} \frac{d \phi}{d x}\right)+Q^{\prime}=0$, where $Q^{\prime}=Q / A d x$ is the volume flow rate per unit volume in units $s^{-1}$. For a constant permeability coefficient, Eq. (6) can be converted to Eq. (7).

$K_{x x} \frac{d^{2} \phi}{d x^{2}}+Q^{\prime}=0$

where the boundary conditions are of the form $\varphi=\varphi_{B}$ on $S_{1}$, $\varphi_{B}$ represents a known boundary fluid flow, and $S_{1}$ is a surface.

For two-dimensional control volumes, as illustrated in Fig. 2, the strong form of the main differential equation can derive analogously. Eq. (8) states the strong form of the fluid flow through a porous medium in the two-dimensional control volume.

$\frac{\partial}{\partial x}\left(K_{x x} \frac{\partial \phi}{\partial x}\right)+\frac{\partial}{\partial y}\left(K_{y y} \frac{\partial \phi}{\partial y}\right)+Q^{\prime}=0$,

with boundary conditions $\varphi=\varphi_{B}$ on $S_{1}$, and

$K_{x x} \frac{\partial \phi}{\partial x} C_{x}+K_{y y} \frac{\partial \phi}{\partial y} C_{y}=$ cons. on $S_{2}$,

where $C_{x}$ and $C_{y}$ are direction cosines of the unit vector normal to the surface $S_{2}$, as illustrated in Fig. 3.

\subsection{The Weak-Form and Finite Element Formulation}

In order to solve Eq. (8), which is known as Laplace's equation, Richard's equation, flow differential equation, etc., its strong form should be converted to a weak form.

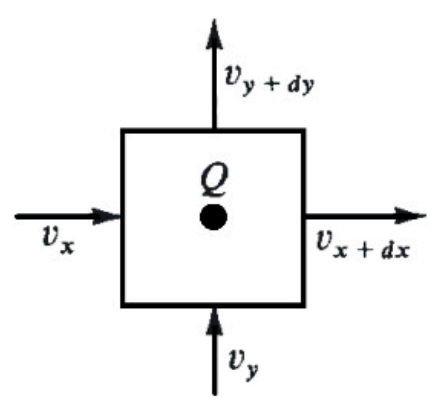

Fig. 2 Control volume for two-dimensional fluid flow

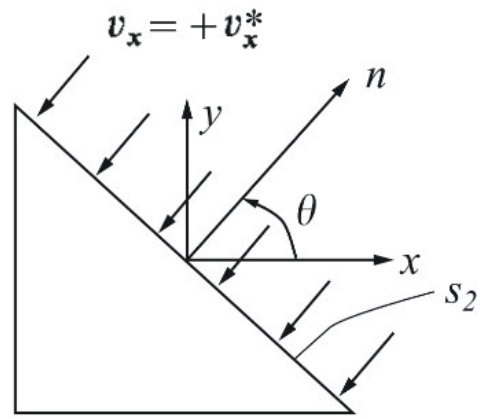

Fig. 3 Unit vector normal to surface $S_{2}$ 
Also, to utilize the finite element formulation in solving procedure, an appropriate element should be considered. In the current study, the three-node triangular element, as illustrated in Fig. 4, is employed to solve the fluid-flow problems in two-dimensional space.

By considering $N$ as the vector of shape functions, the potential function, as Eq. (10), can be stated in terms of nodal potentials.

$[\varphi]=\left[\begin{array}{lll}\boldsymbol{N}_{i} & \boldsymbol{N}_{j} & \boldsymbol{N}_{m}\end{array}\right]\left\{\begin{array}{c}p_{i} \\ p_{j} \\ p_{m}\end{array}\right\}$,

where $p_{i}, p_{j}$, and $p_{m}$ are the nodal potentials. Note that for groundwater flow, $\varphi$ is the piezometric fluid head function. The shape functions can be considered as Eq. (11).

$N_{i}=\frac{1}{2 A}\left(\alpha_{i}+\beta_{i} x+\gamma_{i} y\right)$,

where the $\alpha, \beta$, and $\gamma$ can be calculated using Eqs. (12) to Eq. (14), respectively.

$\alpha_{i}=\left|\begin{array}{ll}x_{j} & x_{m} \\ y_{j} & y_{m}\end{array}\right|, \quad \alpha_{j}=\left|\begin{array}{cc}x_{m} & x_{i} \\ y_{m} & y_{i}\end{array}\right|, \quad \alpha_{m}=\left|\begin{array}{cc}x_{i} & x_{j} \\ y_{i} & y_{j}\end{array}\right|$

$\beta_{i}=y_{j}-y_{m}, \quad \beta_{j}=y_{m}-y_{i}, \quad \beta_{m}=y_{i}-y_{j}$

$\gamma_{i}=x_{m}-x_{j}, \quad \gamma_{j}=x_{i}-x_{m}, \quad \gamma_{m}=x_{j}-x_{i}$

The gradient matrix $g$ can be stated using Eq. (15).

$\{\boldsymbol{g}\}=[\boldsymbol{B}]\{p\}$,

where matrix $\boldsymbol{B}$ is given by Eq. (16).

$[\boldsymbol{B}]=\frac{1}{2 A}\left[\begin{array}{lll}\beta_{i} & \beta_{j} & \beta_{m} \\ \gamma_{i} & \gamma_{j} & \gamma_{m}\end{array}\right]$

Therefore, the gradient matrix $g$ is equal to Eq. (17).

$\{\boldsymbol{g}\}=\left\{\begin{array}{l}\boldsymbol{g}_{x} \\ \boldsymbol{g}_{y}\end{array}\right\}$

Now, the velocity-gradient matrix relationship can be presented as Eq. (18).

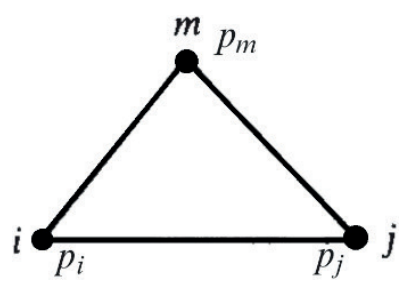

Fig. 4 Fundamental triangular element, including nodal potentials $\left\{\begin{array}{l}v_{x} \\ v_{y}\end{array}\right\}=-[\boldsymbol{D}]\{\boldsymbol{g}\}$,

where the material property matrix, $\boldsymbol{D}$, is defined as Eq. (19).

$[\boldsymbol{D}]=\left[\begin{array}{cc}K_{x x} & 0 \\ 0 & K_{y y}\end{array}\right]$

In the following, the stiffness matrix for each element should be driven. For a fundamental three-node triangular element, the stiffness matrix can be employed as Eq. (20).

$[\boldsymbol{k}]=\iiint_{V}[\boldsymbol{B}]^{T}[\boldsymbol{D}][\boldsymbol{B}] d V$

If the constant-thickness $(t)$ is assumed and noting that the integrated terms are constant, then the Eq. (21) can be used rather than Eq. (20).

$[\boldsymbol{k}]=t A[\boldsymbol{B}]^{T}[\boldsymbol{D}][\boldsymbol{B}]$

The above equation can be simplified to Eq. (22).

$[\boldsymbol{k}]=\frac{t K_{x x}}{4 A}\left[\begin{array}{ccc}\beta_{i}^{2} & \beta_{i} \beta_{j} & \beta_{i} \beta_{m} \\ \beta_{j} \beta_{i} & \beta_{j}^{2} & \beta_{j} \beta_{m} \\ \beta_{m} \beta_{i} & \beta_{m} \beta_{j} & \beta_{m}^{2}\end{array}\right]+\frac{t K_{y y}}{4 A}\left[\begin{array}{ccc}\gamma_{i}^{2} & \gamma_{i} \gamma_{j} & \gamma_{i} \gamma_{m} \\ \gamma_{j} \gamma_{i} & \gamma_{j}^{2} & \gamma_{j} \gamma_{m} \\ \gamma_{m} \gamma_{i} & \gamma_{m} \gamma_{j} & \gamma_{m}^{2}\end{array}\right]$

In dealing with the force matrices, it is possible to define Eq. (23).

$\left\{f_{Q}\right\}=\iiint_{V} Q[\boldsymbol{N}]^{T} d V=Q \iiint_{V}[\boldsymbol{N}]^{T} d V$,

for constant volumetric flow rate per unit volume over the whole element. By using FEM and shape function concepts, Eq. (23) can be converted to Eq. (24).

$\left\{f_{Q}\right\}=\frac{Q V}{3}\left\{\begin{array}{l}1 \\ 1 \\ 1\end{array}\right\}$

Eventually, the second force matrix can be stated as Eqs. (25) and (26).

$$
\begin{aligned}
& \left\{f_{q}\right\}=\iint_{S_{2}} q^{*}[\boldsymbol{N}]^{T} d S=\iint_{S_{2}} q^{*}\left\{\begin{array}{c}
\boldsymbol{N}_{i} \\
\boldsymbol{N}_{j} \\
\boldsymbol{N}_{m}
\end{array}\right\} d S, \\
& \left\{f_{q}\right\}=\frac{q^{*} L_{i-j} t}{2}\left\{\begin{array}{l}
1 \\
1 \\
0
\end{array}\right\},
\end{aligned}
$$

where $L_{i-j}$ is the length of the element, and $q^{*}$ is the assumed constant surface flow rate. 


\section{Optimization algorithms and objective function}

As introduced in Section 1, in this paper, to determine the Critical Failure Surface (CFS), the Factor of Safety (FOS) of the probable CFSs is minimized using a meta-heuristic algorithm. In the current paper, five novel meta-heuristic algorithms are utilized to determine CFS in benchmark problems. All the employed methods contain some mathematical features in their optimizing procedure. Black Hole Mechanics Optimization (BHMO), Enriched Firefly Algorithm (EFA), Covariance Matrix Adaptation Evolution Strategy (CMA-ES), Eigenvectors of the Covariance Matrix (ECM), and Sine Cosine Algorithm (CSA) constitute the set of employed algorithms in this study. In the following, each of them is introduced in a nutshell. Also, at the end of the section, the corresponding objective function with the slope stability problem is presented.

\subsection{Black Hole Mechanics Optimization}

The Black Hole Mechanics Optimization (BHMO) is a newly developed and released meta-heuristic algorithm by Kaveh et al. [19]. The algorithm was inspired by the mechanics of Schwarzschild and Kerr black holes. BHMO employs a robust Mathematical Kernel based on Covariance Matrix formed between each variable and its relative cost. This Covariance Matrix leads to finding the optimum orientation for increasing or decreasing the current variable. By this technique, each variable is directed rapidly towards its relative best value.

Moreover, each variable is assumed independently of the others in comparison with the cost function. This property leads to escaping from the local optimums that are present in the search space of some problems. Besides the Mathematical Kernel, a Physical Simulation helps the conduction of variables in each step. This physical simulation that is based on mentioned black hole Mechanics updates the variables in the vicinity of surmised global best in each step. Also, the elimination of weak variables is due to physical simulation after total navigation by the mathematical kernel. For more computational details, respected readers are referred to [19]. The other well-known metaheuristic used in this paper is PSO that that is taken from Kalatehjari et al. [31].

\subsection{Enriched Firefly Algorithm}

The Firefly Algorithm (FA) is a meta-heuristic algorithm inspired by the flashing behavior of fireflies. There are two critical considerations in the FA. First, the variation of light intensity and second, the formulation of attractiveness.
The appropriate assumption, for simplicity, is that the attractiveness of a firefly is indicated by its brightness that is, in turn, mapped to the encoded cost function. In minimization cases, the brightness of a firefly at a location can be selected approximately.

The basic version of the Firefly algorithm (FA) was presented by Yang [32] and has been applied successfully in either continuous or discrete optimization problems. Although it is proved that FA is a better algorithm than many other optimization meta-heuristic algorithms [33], however, there are some drawbacks in its computational processes that increase the FA computational complexity. For instance, Mai [34] indicated that the FA could not found the optimum solution in some problems and that it was trapped into the local optima. Therefore, Kaveh and Ilchi Ghazaan [35] proposed an Enriched Firefly Algorithm (EFA) in which by some minor tricks, the robustness of the basic FA is increased.

\subsection{Covariance Matrix Adaptation Evolution Strategy}

The Covariance Matrix Adaptation Evolution Strategy (CMA-ES) is a novel-mathematical-based meta-heuristic algorithm that is proposed by Hansen [20]. The CMA-ES is a particular type of strategy for numerical optimization in which two main principles are considered for the adaptation of parameters of the search space distribution. Firstly, calculating the Maximum-Likelihood principle to increase the probability of successful candidate solution and search iterations. Secondly, recording two paths of the time evolution of the distribution mean of the strategy to contain relevant data about the correlation between consecutive iterations. Many meta-heuristic algorithms, such as BHMO, ECM, etc., are affected by the principal idea of the CMA-ES. Reference [20] includes an appropriate review of the Covariance Matrix Adaptation Evolution Strategy algorithm.

\subsection{Eigenvectors of the Covariance Matrix}

Pouriyanezhad et al. [21], by combining eigenvectors of the covariance matrix and random normal distribution, proposed a new method meta-heuristic method. The main idea of the Eigenvector Covariance Matrix (ECM) algorithm is due to the CMA-ES method. The ECM generates some initial random solutions in each iteration, then by employing a dynamic penalty function assigns a value to the solutions. The most novelty in the ECM is to consider the least violated data as the desired one and employ the corresponding covariance matrix with the desire solutions 
to conduct and improve initial solutions. This new and novel algorithm includes high performance, especially in structural engineering problems.

\subsection{Sine Cosine Algorithm}

The Sine Cosine Algorithm (SCA) is a novel population-based meta-heuristic method that can be categorized as mathematical-based algorithms. The SCA is proposed by Mirjalili in which a set of initial random solutions is generated. Then the initial solutions are improved using trigonometry equations. There are some stochastic parameters in the SCA that play vital roles in its performance. According to a fluctuation behavior, the initial solutions converge to the global bests. Another algorithm in which the fluctuation behavior is utilized to optimize problems is the Vibrating Particles System proposed by Kaveh and Ilchi Ghazaan [35]. The SCA can obtain optimal solutions in continuous problems. The most important note is that the problem should be unconstrained with one objective function.

\subsection{Objective function}

As introduced previously, the most appropriate CFS is one contains the minimum corresponding FOS. In the current study, Bishop's method, based on the Limit Equilibrium Technique (LET), is employed to obtain the Factor of Safety of slopes against failure. Generally, a Factor of Safety (FOS) can be defined as Eq. (27).

$$
F O S=\frac{\sum S_{r e s .},}{\sum S_{m o b .}},
$$

where $S_{\text {resistance }}$ and $S_{\text {mobilized }}$ can be defined as Eq. (28) and Eq. (29), respectively.

$$
\begin{aligned}
& S_{\text {res. }}=c^{\prime}+(N-U) \tan \phi^{\prime}, \\
& S_{\text {mob. }}=W \sin \alpha,
\end{aligned}
$$

herein, $c^{\prime}$ is the effective cohesion, $N$ is base normal force and is equal to $N=W \cos \alpha, U$ is the total pore-water pressure, $\varphi^{\prime}$ is the effective frictional angle, $W$ is the slice weight, and $\alpha$ is base inclination.

By considering a seismic pseudo-static stability analysis of slopes and applying an acceleration that creates inertia forces, Eqs. (30) and (31) can be defined.

$$
\begin{aligned}
& F_{h}=\left(a_{h} W / g\right)=k_{h} W, \\
& F_{v}=\left(a_{v} W / g\right)=k_{v} W,
\end{aligned}
$$

where subscripts $h$ and $v$ indicate the effect in horizontal and vertical, respectively, also, $F, a$, and $g$ represent force, acceleration, and gravitational acceleration, sequentially.

Eventually, the Factor of Safety (FOS) equation under lateral pseudo-static earthquake acceleration using Bishop's method, as the objective function, can be calculated using Eq. (32).

$$
f=\frac{\sum_{1}^{n s l i c e}\left[c^{\prime} l+\left(W-\frac{c^{\prime} l \sin \alpha}{f}-U \cos \alpha-F_{h} \sin \alpha\right) \tan \phi^{\prime}\right] \frac{1}{m_{\alpha}}}{\sum_{1}^{n s l i c e}\left(W \sin \alpha+F_{h} \cos \alpha\right)}
$$

where $l$ is slice base length, $n_{\text {slice }}$ is the number of slices, and $m_{\alpha}$ can be calculated using Eq. (33).

$m_{\alpha}=\cos \alpha+\frac{\sin \alpha \tan \phi^{\prime}}{f}$

Therefore, the CFS determination aims to minimize Eq. (32) by changing the position of center of CFS and its corresponding radius within the search space or slope.

\section{Numerical examples}

The current section is dedicated to determining the CFS of the benchmark slopes using introduced meta-heuristic algorithms. For this purpose, firstly, some random solutions are generated based on each algorithm approach. Each answer contains three individual data: $x$ and $y$ coordinates of the CFS center and its radius, respectively. Then, the slope geometry should be divided into some slices. This partitioning is due to the cross points of the CFS and slope geometry. After that, an appropriate FEM mesh should be generated to obtain slice parameters, such as weight, pore-water pressure, etc., using finite element analysis. Herein, the objective function, i.e., Factor of Safety (FOS), should be evaluated. By repeating this procedure, the optimum position of the CFS and minimum FOS will be obtained. For simplicity, the CFS is considered a circular, and Fixed Slice Division Method (FSDM) is employed. The introduced procedure is illustrated in Fig. 5.

In the following, the optimization procedure for obtaining Critical Failure Surface (CFS) is utilized to solve two geotechnical benchmark problems.

\subsection{Benchmark problem I}

The first example, as illustrated in Fig. 6, includes a homogenous soil slope investigated previously by Malkawai et al. [24]. In this example, the geotechnical parameters 


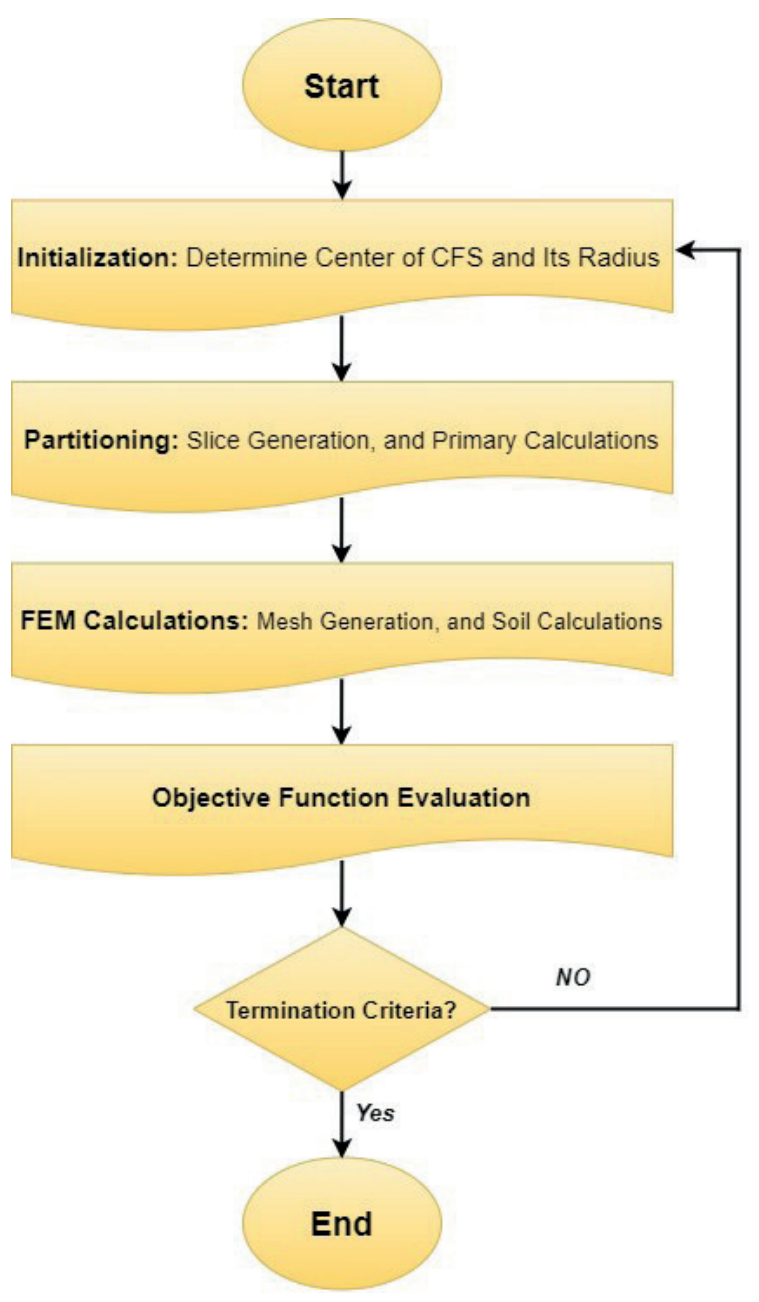

Fig. 5 The corresponding flowchart with the optimization procedure of CFS determination

are as follows: effective cohesion $c^{\prime}=9.8 \mathrm{kN} / \mathrm{m}^{2}$, angle of internal friction $\varphi^{\prime}=10$ degrees, and unit weight $\gamma=17.64 \mathrm{kN} / \mathrm{m}^{3}$.

The slope has been analyzed using the introduced optimization procedure in Section 3. The population size of each algorithm is considered as $N=40$, and the maximum number of iterations as $I T=60$. To compare the final obtained results by different algorithms, each metaheuristic method solved the problem 30 times. Then the mean of the solutions is considered as the performance of the employed method. Also, the number of slices is regarded as 20. Fig. 7 illustrates the obtained CFSs for benchmark problem 1.

As illustrated in Fig. 7, since all utilized meta-heuristic algorithms are robust and powerful, they could optimize the problem and obtain appropriate Critical Failure Surface (CFS). However, there are some differences in their procedure and results that are discussed in the following. The statistical results of the obtained CFSs and the final solution of each algorithm are reported in Table 1

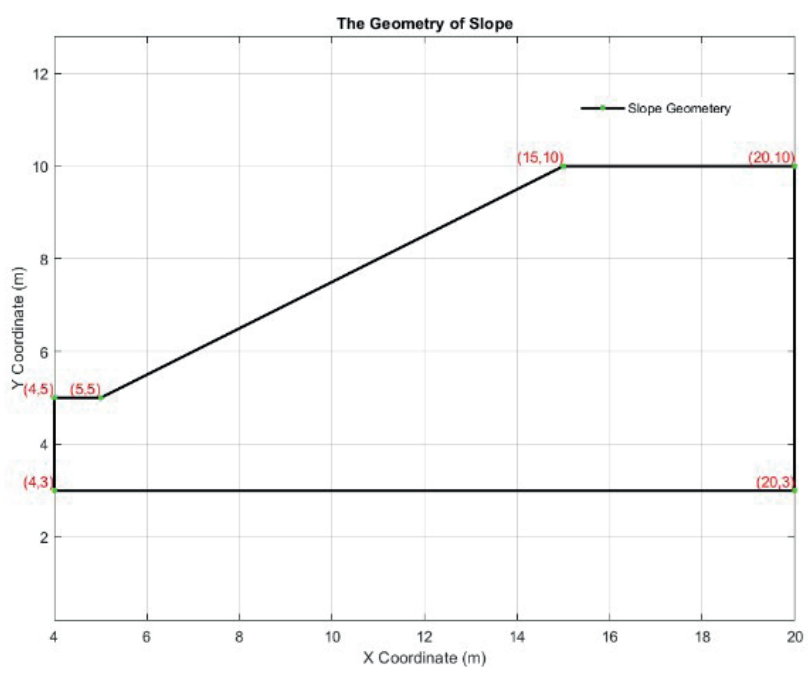

Fig. 6 The geometry of the first benchmark slope

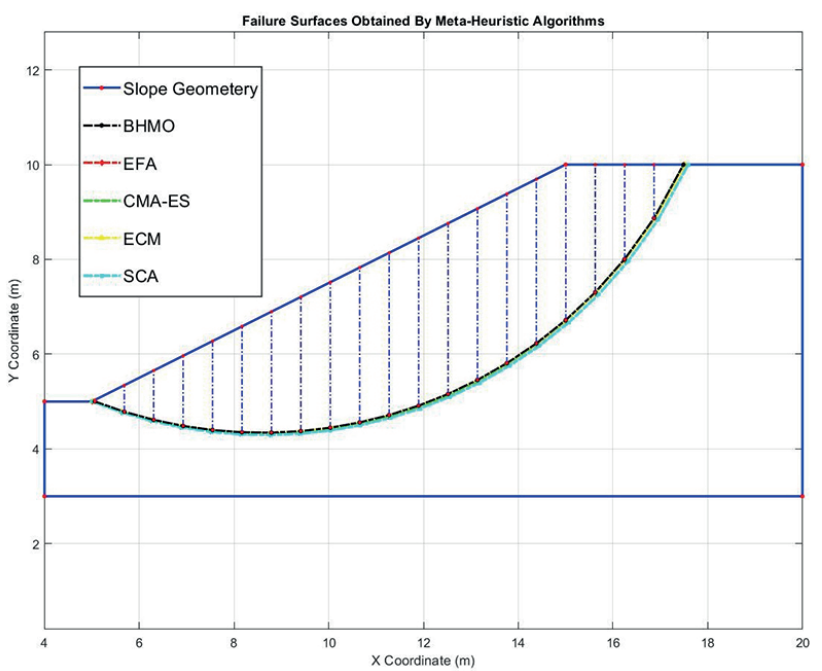

(a)

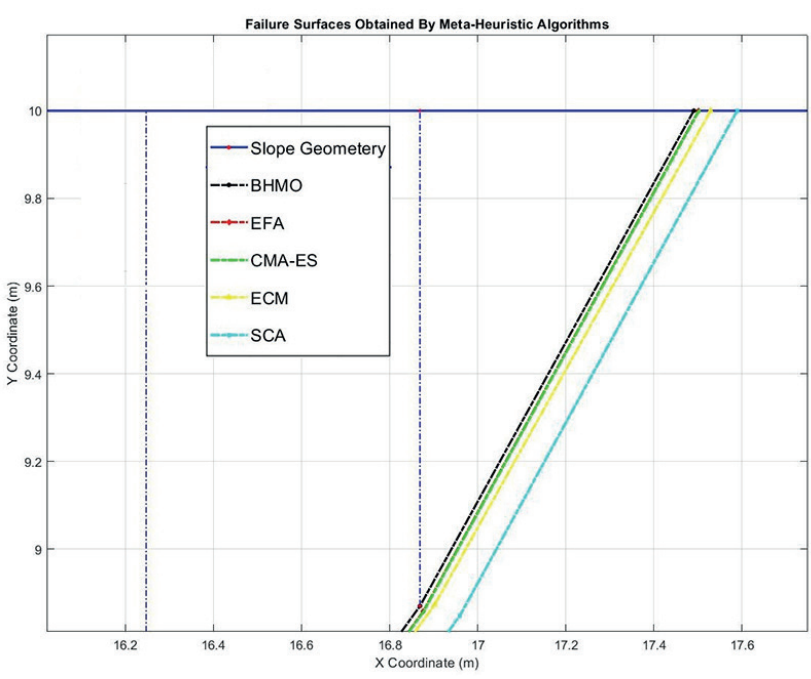

(b)

Fig. 7 The critical failure surfaces obtained by different meta-heuristic algorithms; a) overall view, b) details on the right cross point 
Table 1 The statistical results of the first benchmark analysis

\begin{tabular}{lcccccc}
\hline & & BHMO & EFA & CMA-ES & ECM & SCA \\
\hline \multirow{3}{*}{ Benchmark 1 } & Best & $1.30 \mathrm{E}+00$ & $1.31 \mathrm{E}+00$ & $1.73 \mathrm{E}+00$ & $1.32 \mathrm{E}+00$ & $2.13 \mathrm{E}+00$ \\
& Average & $1.30 \mathrm{E}+00$ & $1.31 \mathrm{E}+00$ & $2.17 \mathrm{E}+00$ & $1.33 \mathrm{E}+00$ & $2.85 \mathrm{E}+00$ \\
& Std. & $9.20 \mathrm{E}-14$ & $4.28 \mathrm{E}-03$ & $5.75 \mathrm{E}-01$ & $2.41 \mathrm{E}-02$ & $4.69 \mathrm{E}-01$ \\
\hline
\end{tabular}

Table 2 The final solutions determined by the meta-heuristic algorithms

\begin{tabular}{lccccc}
\hline CFS Properties & BHMO & EFA & CMA-ES & ECM & SCA \\
\hline x Coordinate & 8.5962 & 8.5964 & 8.6080 & 8.5767 & 8.6624 \\
y Coordinate & 14.1563 & 14.1325 & 14.1291 & 14.2398 & 14.1322 \\
Radius & 9.8345 & 9.8320 & 9.8412 & 9.9175 & 9.8613 \\
\hline
\end{tabular}

Table 3 The FOS value of the benchmark problem 1

\begin{tabular}{lcccc}
\hline Researcher & Method & Number of Slices & Limit Equilibrium Method & FOS \\
\hline Yamagami and Veta [36] & BFGS & - & Morgenstern-Price Method & 1.3380 \\
Yamagami and Veta [36] & DFP & - & Morgenstern-Price Method & 1.3380 \\
Yamagami and Veta [36] & Powell & - & Morgenstern-Price Method & 1.3380 \\
Yamagami and Veta [36] & Nelder-Mead & - & Morgenstern-Price Method & 1.3480 \\
Greco [37] & Pattern Search & - & Spencer's Method & 1.3300 \\
Greco [37] & Monte Carlo & - & Spencer's Method & 1.3330 \\
Malkawai et al. [24] & Monte Carlo & - & Spencer's Method & 1.2380 \\
Cheng et al. [38] & PSO & 20 & Spencer's Method & 1.3285 \\
Kalatehjari et al. [31] & PSO & 24 & Bishop's Method & 1.3128 \\
Himanshu and Burman [39] & PSO & 25 & Bishop's Method & 1.3141 \\
Present study & BHMO & 20 & Bishop's Method & 1.3044 \\
Present study & EFA & 20 & Bishop's Method & 1.3140 \\
Present study & CMA-ES & 20 & Bishop's Method & 1.7289 \\
Present study & ECM & 20 & Bishop's Method & 1.3207 \\
Present study & SCA & 20 & Bishop's Method & 2.1335
\end{tabular}

and Table 2, respectively. Also, the obtained results are compared to other efforts, as detailed in Table 3. Finally, the optimization procedure is shown in Fig. 8.

As reported in Table 3, meta-heuristics has been applied to this benchmark problem successfully. According to the type of the current problem and its mathematical principles, it seems that those of mathematically based meta-heuristics should be more appropriate to employ as the optimization method. Therefore, in this paper, all employed meta-heuristics are mathematically based. Through this type of algorithms, according to Table 1, it seems that those of methods that use statistical concepts, such as covariance matrix, in their procedure are more appropriate to solve this type of problem (i.e., structural and geotechnical problems). This may be due to the logical background of the engineering problems that may be modeled more suitable by mathematical based algorithms.

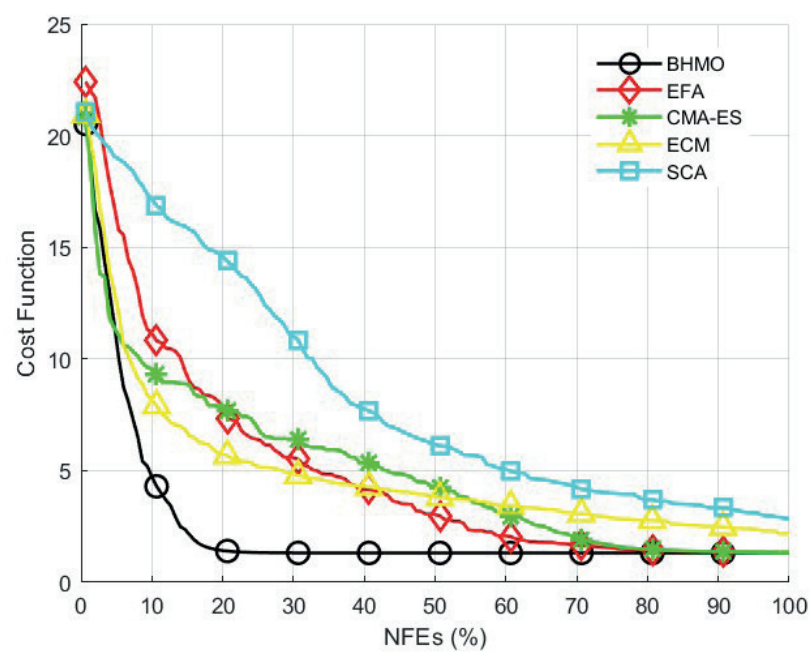

Fig. 8 The optimization procedure by different meta-heuristic algorithms (benchmark problem 1) 


\subsection{Benchmark problem II}

The second benchmark problem investigated in the current study has been taken from the effort of Zolfaghari et al. [25]. The studied slope contains a homogenous soil slope, and the geometric layout for the soil slope is illustrated in Fig. 9.

For the mentioned slope, the geotechnical properties are as follows: effective cohesion $c^{\prime}=14.71 \mathrm{kN} / \mathrm{m}^{2}$, angle of internal friction $\varphi^{\prime}=20$ degrees, and unit weight $\gamma=18.63 \mathrm{kN} / \mathrm{m}^{3}$. Other computational details are similar to the benchmark problem 1. Fig. 10 illustrates the obtained CFS by meta-heuristic algorithms. The optimization procedure is shown in Fig. 11, finally, Tables 4 to 6 report the statistical and comparative results of the considered problem.

According to the reported data, it is possible to say that the related concluded remarks to the first example might be mentioned again. There is an important note that the population size, maximum number of iterations, and number of slices is decidedly smaller than other methods specified in Table 6. It is obvious that if these algorithm parameters (i.e., the maximum number of iterations, population size, and the number of slices) is increased, then all the employed methods will achieve the best solution due to their procedure. However, in comparison with the mentioned method in Table 6 , by less computational costs, the utilized algorithm could achieve accepted results. This property is due to its robustness and its mathematical conductivity of initial solutions. In this study, among employed meta-heuristic methods, the Black Hole Mechanics Optimization (BHMO) algorithm, contains the highest performance. This performance may be due to its procedure in which the covariance matrix is employed several to conduct initial solutions to the best one. Another note that affects the

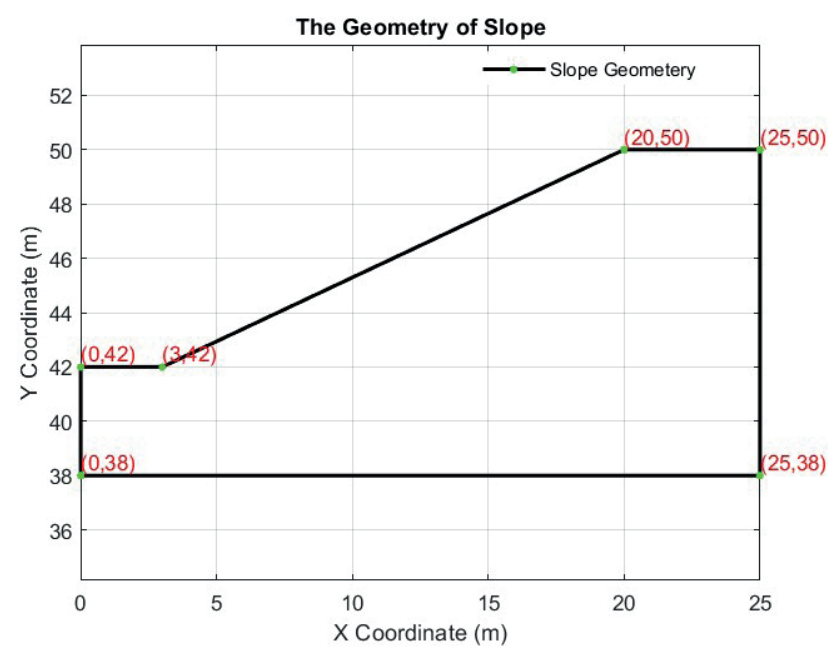

Fig. 9 The geometry of the second benchmark slope

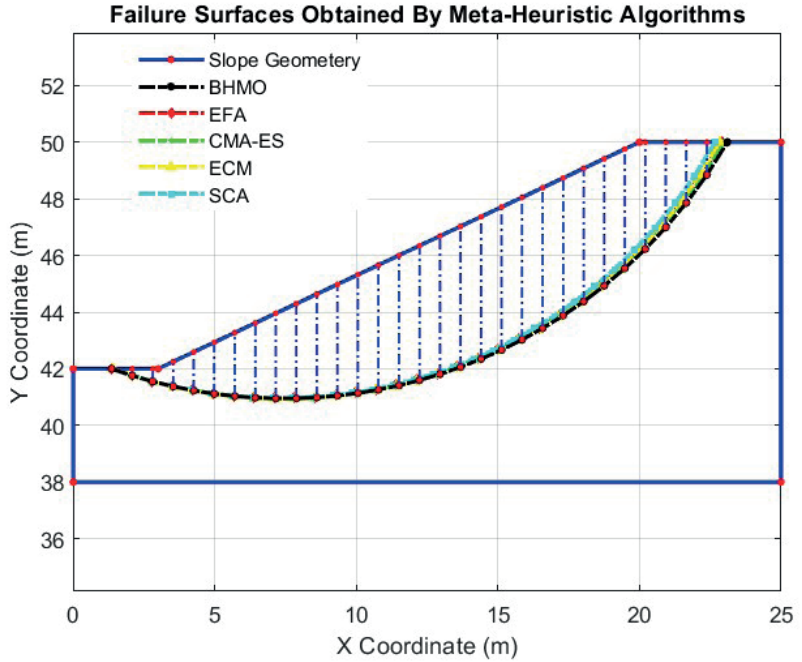

(a)

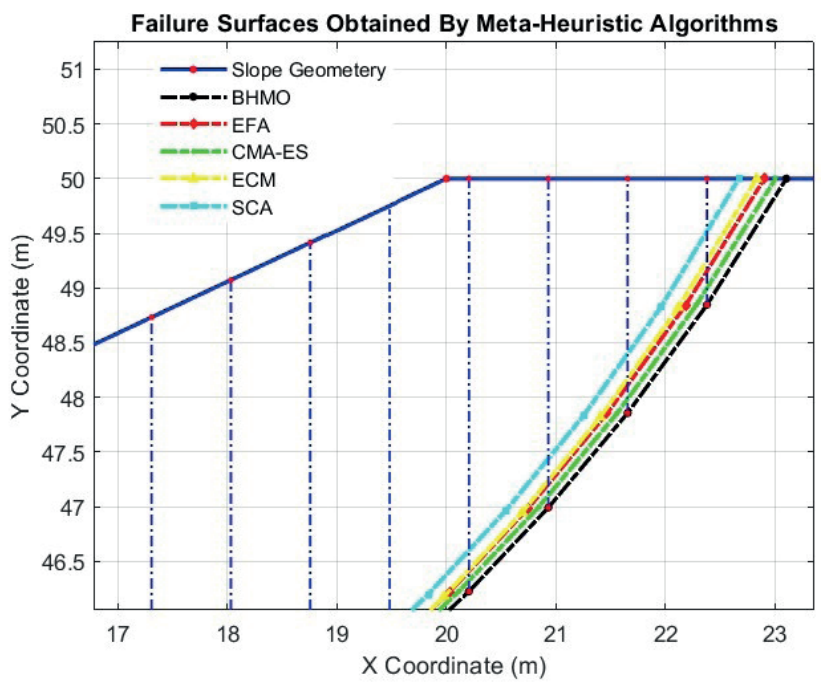

(b)

Fig. 10 The critical failure surfaces obtained by different meta-heuristic algorithms; a) overall view, b) details on the right cross point

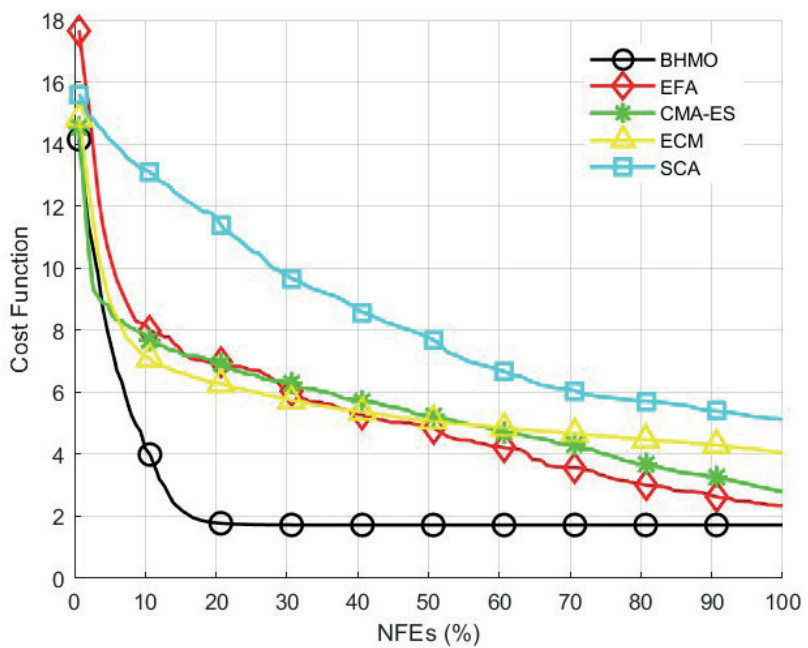

Fig. 11 The optimization procedure by different meta-heuristic algorithms (benchmark problem 2) 
Table 4 The statistical results of the second benchmark analysis

\begin{tabular}{lcccccc}
\hline & & BHMO & EFA & CMA-ES & ECM \\
\hline \multirow{3}{*}{ Benchmark 2 } & Best & $1.71 \mathrm{E}+00$ & $1.72 \mathrm{E}+00$ & $1.94 \mathrm{E}+00$ & $1.84 \mathrm{E}+00$ & $1.98 \mathrm{E}+00$ \\
& Average & $1.72 \mathrm{E}+00$ & $1.74 \mathrm{E}+00$ & $1.98 \mathrm{E}+00$ & $1.80 \mathrm{E}+00$ & $2.10 \mathrm{E}+00$ \\
& Std. & $1.92 \mathrm{E}-12$ & $1.74 \mathrm{E}-01$ & $3.55 \mathrm{E}-01$ & $3.60 \mathrm{E}-01$ & $4.71 \mathrm{E}-01$ \\
\hline
\end{tabular}

Table 5 The final solutions determined by the meta-heuristic algorithms

\begin{tabular}{|c|c|c|c|c|c|}
\hline CFS Properties & BHMO & EFA & CMA-ES & $\mathrm{ECM}$ & $\mathrm{SCA}$ \\
\hline x Coordinate & 7.4386 & 7.4184 & 7.4057 & 7.7538 & 7.3752 \\
\hline y Coordinate & 59.0521 & 58.8634 & 58.8734 & 58.6321 & 58.5241 \\
\hline Radius & 18.1049 & 17.8615 & 18.0298 & 17.6034 & 17.5943 \\
\hline
\end{tabular}

Table 6 The FOS value of the benchmark problem 2

\begin{tabular}{lccc}
\hline Researcher & Method & Number of Slices & Limit Equilibrium Method \\
\hline Zolfaghari et al. [25] & GA & - & Bishop's Method \\
Zolfaghari et al. [25] & GA & - & Morgenstern Method \\
Zolfaghari et al. [25] & GA & - & Morgenstern Method \\
Cheng et al. [38] & PSO & 40 & Spencer's Method \\
Kalatehjari et al. [31] & PSO & 40 & Bishop's Method \\
Himanshu and Burman [39] & PSO & 25 & Bishop's Method \\
Present study & BHMO & 20 & Bishop's Method \\
Present study & EFA & 20 & Bishop's Method \\
Present study & CMA-ES & 20 & Bishop's Method \\
Present study & ECM & 20 & Bishop's Method \\
Present study & SCA & 20 & Bishop's Method \\
\hline
\end{tabular}

efficiency of the utilized optimization procedure in the current study is the FEM employment. The utilization of the FEM helps the procedure to obtain FOS more accurately than other approximately approaches.

\section{Conclusions}

In this paper, five robust meta-heuristic algorithms are utilized to optimize the slope stability problem. In order to obtain the Critical Failure Surface (CFS) and its corresponding Factor of Safety (FOS), the Finite Element Method (FEM) is employed. In addition to the general loading, seismic forces and seepage effect are considered, as well. The results are compared with other efforts mentioned in the literature. According to the results, it can be deducted that those of meta-heuristic methods which contain some mathematical principles in their optimization

\section{References}

[1] Fellenius, W. "Calculation of stability of earth dam", In: Transactions, 2nd International Congress on Large Dams, Washington, DC, USA, 1936, pp. 445-462.

[2] Bishop, A. W., Morgenstern, N. R. "Stability coefficients for earth slopes", Géotechnique, 10(4), pp. 129-153, 1960.

https://doi.org/10.7939/R35M62D9F procedure, probably are more successful in dealing with the current geotechnical problem. Therefore, all selected meta-heuristic methods in the present study contain some mathematical steps in their main algorithm.

Among utilized meta-heuristic algorithms (all of them include mathematical theories), those executing statistical concepts, such as the covariance matrix among some variables, are more successful in optimizing benchmark problems. Based on statistical reports, it seems that the Black Hole Mechanics Optimization (BHMO) algorithm is more suitable in solving the slope stability problem. This can be due to the several utilization of the statistical concepts.

\section{Compliance with ethical standards}

Conflict of interest: No potential conflict of interest was reported by the authors.

[3] Morgenstern, N. R., Price, V. E. "The Analysis of the Stability of General Slip Surfaces", Géotechnique, 15(1), pp. 79-93, 1965. https://doi.org/10.1680/geot.1965.15.1.79

[4] Spencer, E. "A Method of analysis of the Stability of Embankments Assuming Parallel Inter-Slice Forces", Géotechnique, 17(1), pp. 11-26, 1967.

https://doi.org/10.1680/geot.1967.17.1.11 
[5] Baker, R., Garber, M. "Theoretical analysis of the stability of slopes", Géotechnique, 28(4), pp. 395-411, 1978.

https://doi.org/10.1680/geot.1978.28.4.395

[6] Chen, Z.-Y., Shao, C.-M. "Evaluation of minimum factor of safety in slope stability analysis", Canadian Geotechnical Journal, 25(4), pp. 735-748, 1988.

https://doi.org/10.1139/t88-084

[7] Arai, K., Tagyo, K. "Determination of Noncircular Slip Surface Giving the Minimum Factor of Safety in Slope Stability Analysis", Soils and Foundations, 25(1), pp. 43-51, 1985.

https://doi.org/10.3208/sandf1972.25.43

[8] He, L., Gomes, A. T., Broggi, M., Beer, M. "Failure Analysis of Soil Slopes with Advanced Bayesian Networks", Periodica Polytechnica Civil Engineering, 63(3), pp. 763-774, 2019.

https://doi.org/10.3311/ppci.14092

[9] Varga, G., Czap, Z. "Soil models: safety factors and settlements", Periodica Polytechnica Civil Engineering, 48(1-2), pp. 53-63, 2004. [online] Available at: https://pp.bme.hu/ci/article/view/582

[10] Kaveh, A. "Advances in Metaheuristic Algorithms for Optimal Design of Structures", 2nd ed., Springer, Cham, Switzerland, 2017. https://doi.org/10.1007/978-3-319-05549-7

[11] Coello, C. C., Lamont, G. B., van Veldhuizen, D. A. "Evolutionary Algorithms for Solving Multi-Objective Problems", 2nd ed., Springer, Boston, MA, USA, 2007. https://doi.org/10.1007/978-0-387-36797-2

[12] Kennedy, J., Eberhart, R. "Particle swarm optimization", In: Proceedings of ICNN'95-International Conference on Neural Networks, Perth, WA, Australia, 1995, pp. 1942-1948. https://doi.org/10.1109/ICNN.1995.488968

[13] Dorigo, M., Birattari, M., Stützle, T. "Ant Colony Optimization", IEEE Computational Intelligence Magazine, 1(4), pp. 28-39, 2006. https://doi.org/10.1109/ci-m.2006.248054

[14] Kaveh, A., Seddighian, M. R. "Simultaneously multi-material layout, and connectivity optimization of truss structures via an Enriched Firefly Algorithm", Structures, 27, pp. 2217-2231, 2020. https://doi.org/10.1016/j.istruc.2020.07.029

[15] Goldberg, D. "Competent Probabilistic Model Building Genetic Algorithms", Department of General Engineering, The University of Illinois at Urbana-Champaign, Urbana, IL, USA, Rep. F4962000-1-0163, India, 2006.

https://doi.org/10.21236/ada416564

[16] Mirjalili, S. "Evolutionary Algorithms and Neural Networks", Springer, Cham, Switzerland, 2019. https://doi.org/10.1007/978-3-319-93025-1

[17] Kaveh, A., Mahdavi, V. R. "Colliding bodies optimization: A novel meta-heuristic method", Computers \& Structures, 139, pp. 18-27, 2014. https://doi.org/10.1016/j.compstruc.2014.04.005

[18] Kaveh, A., Dadras, A. "A novel meta-heuristic optimization algorithm: Thermal exchange optimization", Advances in Engineering Software, 110, pp. 69-84, 2017. https://doi.org/10.1016/j.advengsoft.2017.03.014

[19] Kaveh, A., Seddighian, M. R., Ghanadpour, E. "Black Hole Mechanics Optimization: a novel meta-heuristic algorithm", Asian Journal of Civil Engineering, 21, pp. 1129-1149, 2020. https://doi.org/10.1007/s42107-020-00282-8
[20] Hansen, N. "The CMA Evolution Strategy: a Comparing Review", In: Lozano, J. A., Larrañaga, P., Inza, I., Bengoetxea, E. (eds.) Towards a New Evolutionary Computation, Springer, Berlin, Heidelberg, Germany, 2006, pp. 75-102. https://doi.org/10.1007/11007937_4

[21] Pouriyanezhad, E., Rahami, H., Mirhosseini, S. M. "Truss optimization using eigenvectors of the covariance matrix", Engineering with Computers, 2020 https://doi.org/10.1007/s00366-020-00943-x

[22] Simon, D. "Biogeography-Based Optimization", IEEE Transactions on Evolutionary Computation, 12(6), pp. 702-713, 2008. https://doi.org/10.1109/tevc.2008.919004

[23] Wolpert, D. H., Macready, W. G. "No free lunch theorems for optimization", IEEE Transactions on Evolutionary Computation, 1(1), pp. 67-82, 1997.

https://doi.org/10.1109/4235.585893

[24] Malkawi, A. I. H., Hassan, W. F., Sarma, S. K. "Global Search Method for Locating General Slip Surface Using Monte Carlo Techniques", Journal of Geotechnical and Geoenvironmental Engineering, 127(8), pp. 688-698, 2001. https://doi.org/10.1061/(asce)1090-0241(2001)127:8(688)

[25] Zolfaghari, A. R., Heath, A. C., McCombie, P. F. "Simple genetic algorithm search for critical non-circular failure surface in slope stability analysis", Computers and Geotechnics, 32(3), pp. 139-152, 2005 .

https://doi.org/10.1016/j.compgeo.2005.02.001

[26] Movahedi Rad, M. "Reliability Based Analysis and Optimum Design of Laterally Loaded Piles", Periodica Polytechnica Civil Engineering, 61(3), pp. 491-497, 2017. https://doi.org/10.3311/PPci.8756

[27] Lógó, J., Movahedi Rad, M., Knabel, J., Tauzowski, P. "Reliability based design of frames with limited residual strain energy capacity", Periodica Polytechnica Civil Engineering, 55(1), pp. 13-20, 2011. https://doi.org/10.3311/pp.ci.2011-1.02

[28] Kaveh, A., Seddighian, M. R., Sadeghi, H., Sadat Naseri, S. "Optimal solution of Richards' equation for slope instability analysis using an integrated enhanced version of black hole", International Journal of Optimization in Civil Engineering, 10(4), pp. 629-650, 2020. [online] Available at: http://ijoce.iust.ac.ir/article-1-456-en.html

[29] Tauzowski, P., Blachowski, B., Lógó, J. "Topology optimization of elasto-plastic structures under reliability constraints: A first order approach", Computers and Structures, 243, Article number: 106406, 2021.

https://doi.org/10.1016/j.compstruc.2020.106406

[30] Blachowski, B., Tauzowski, P., Lógó, J. "Yield limited optimal topology design of elastoplastic structures", Structural Multidisciplinary Optimization, 61, pp. 1953-1976, 2020.

https://doi.org/10.1007/s00158-019-02447-9

[31] Kalatehjari, R., Ali, N. B., Ashrafi, E. "Finding the critical slip surface of a soil slope with the aid of particle swarm optimization", International Multidisciplinary Scientific GeoConference: SGEM, 1(1), pp. 459-466, 2011.

https://doi.org/10.5593/sgem2011/s02.118 
[32] Yang, X.-S. "Firefly Algorithms for Multimodal Optimization", In: Stochastic Algorithms: Foundations and Applications, Proceedings of the 5th International Symposium, SAGA 2009, Sapporo, Japan, 2009, pp. 169-178.

[33] Strumberger, I., Bacanin, N., Tuba, M. "Enhanced firefly algorithm for constrained numerical optimization", In: 2017 IEEE Congress on Evolutionary Computation (CEC), San Sebastian, Spain, 2017, pp. 2120-2127.

https://doi.org/10.1109/cec.2017.7969561

[34] Mai, G. "Application of firefly algorithm and its parameter setting for job shop scheduling", In: Advances in Engineering Research, Proceedings of the 2016 4th International Conference on Machinery, Materials and Computing Technology, Hangzhou, China, 2012, pp. 1658-1662.

https://doi.org/10.2991/icmmct-16.2016.330

[35] Kaveh, A., Ilchi Ghazaan, M. "Vibrating particles system algorithm for truss optimization with multiple natural frequency constraints", Acta Mechanica, 228(1), pp. 307-322, 2017.

https://doi.org/10.1007/s00707-016-1725-z
[36] Yamagami, T., Veta, Y. "Search for noncircular slip surfaces by the Morgenstern-Price method", In: Proceedings of the Sixth International Conference on Numerical Methods in Geomechanics, Innsbruck, Austria, 1988, pp. 1335-1340.

[37] Greco, V. R. "Efficient Monte Carlo technique for locating critical slip surface", Journal of Geotechnical Engineering, 122(7), pp. 517$525,1996$. https://doi.org/10.1061/(asce)0733-9410(1996)122:7(517)

[38] Cheng, Y. M., Li, L., Chi, S., Wei, W. B. "Particle swarm optimization algorithm for the location of the critical non-circular failure surface in two-dimensional slope stability analysis", Computers and Geotechnics, 34(2), pp. 92-103, 2007.

https://doi.org/10.1016/j.compgeo.2006.10.012

[39] Himanshu, N., Burman, A. "Determination of Critical Failure Surface of Slopes Using Particle Swarm Optimization Technique Considering Seepage and Seismic Loading", Geotechnical and Geological Engineering, 37(3), pp. 1261-1281, 2019.

https:/doi.org/10.1007/s10706-018-0683-8 